\title{
Caracterización de pacientes pediátricos con enfermedad de Von Willebrand en un centro de referencia en Bogotá (Colombia)
}

\author{
A. Linares, S. Castaño, I. C. Sarmiento, J. M. Cortés, E. Beltrán, E. Cabrera, F. Ortiz
}

Oncohematólogos pediatras, Fundación Hospital de la Misericordia (HOMI),

Universidad Nacional de Colombia.

\begin{abstract}
Introducción. La enfermedad de Von Willebrand es el trastorno de la coagulación más frecuente, las manifestaciones clínicas son variables, la mayoría de las veces leves-moderadas.

Objetivo. Describir las características clínicas y de laboratorio de $<18$ años con diagnóstico de VWB en un centro de referencia.

Método. Se realizó un estudio de serie de casos con recolección retrospectiva de datos, se definió caso como paciente con clínica y resultados de laboratorio de VWB (TPT, factor VIII, antígeno de VW, cofactor de ristocetina y curvas de agregación plaquetaria).

Resultados. Se analizaron los datos de 32 pacientes, el 59,3\% masculinos, con un promedio de edad de 9,3 años (DE: 5,01). Las manifestaciones de sangrado fueron epistaxis (16/25), gingivorragia (8/25), menorragia (5/25). El 85\% de los pacientes tenían el TPT prolongado, se encontraron 12/32 con tipo I (deficiencia de antígeno de Von Willebrand), 18/32 tipo 2 (alteración funcional de Von Willebrand) y 2/32 tipo III (ausencia de antígeno de Von Willebrand). El tratamiento que con mayor frecuencia requirieron los pacientes fue concentrado de factor VIII/NW derivado plasmático (11/13) y desmopresina (2/13). Catorce pacientes tienen antecedentes en la familia de trastorno de la coagulación.
\end{abstract}

Conclusiones. Se encontró un porcentaje mayor de pacientes con EVW tipo 2, no es clara la razón. Es necesario considerar el diagnóstico de VWB en los pacientes con manifestaciones hemorrágicas.

\section{Estudio molecular de la inversión de los intrones 1 y 22 del factor VIII de la coagulación en un grupo de niños con diagnóstico de hemofilia A en Colombia}

\author{
M. F. Garcés ${ }^{1}$, A. Linares $1,2,3$, I. C. Sarmiento 3 , J. E. Caminos¹, S. Castaño 1,3 , M. Cortés ${ }^{3}$
}

${ }^{1}$ Universidad Nacional de Colombia.

${ }^{2}$ Clínica Infantil Colsubsidio.

${ }^{3}$ Fundación Hospital de la Misericordia.

Introducción. La hemofilia A es una enfermedad recesiva ligada al cromosoma $X$, que presenta una incidencia de 1 en 5.000-10.000 varones, lo que la convierte en uno de los desórdenes más comunes de la hemostasia. La inversión del intrón 22 se considera la alteración más frecuente, con una prevalencia del $45 \%$ al $50 \%$, seguida de la inversión en el intrón 1, con una prevalencia del $0,3 \%$ al $5 \%$ de los pacientes con fenotipo severo.

Objetivo. Estudiar la inversión de los intrones 1 y 22 (INV1, INV22) del gen del factor VIII de la coagulación, mediante técnicas de LD $P C R$, en un grupo de pacientes pediátricos con diagnóstico de hemofilia A que asisten al servicio de hematología de diferentes IPS en Bogotá, D.C. (Colombia)
Material y métodos. Estudio no experimental, descriptivo, transversal.

Resultados. En el presente estudio se analizó por primera vez la frecuencia de las dos mutaciones más frecuentes en hemofilia A severa en una cohorte de 30 pacientes pediátricos colombianos. Se detectó la INV22 en el $40 \%$ y la INV1 en el $10 \%$ de la población estudiada. En los pacientes en quienes se detectó la INV22, el 33\% han desarrollado inhibidores.

Conclusiones. El hallazgo de la frecuencia de la INV22 es similar a los reportados por la literatura en otros grupos de población con diagnóstico de hemofilia A severa. 